\title{
Modeling Steady-state Foam Flow: Hysteresis and Backward Front Movement
}

\author{
Muhammad M. Almajid,* Negar Nazari,* and Anthony R. Kovscek* \\ Energy Resources Engineering, Stanford University, 367 Panama St., Room 050, Stanford, \\ CA 94305, USA
}

E-mail: majimm0a@stanford.edu; negar.nazari@stanford.edu; kovscek@stanford.edu

\section{Supporting Information}

This supporting information details the development of the steady-state model in the main text as well as presents validation and verification of the model.

\section{Development of the Steady-state Population Balance Model}

Inserting the rate of generation and coalescence into the full-physics, steady-state, population balance model in $1 D$ (Eq. 3 in the main manuscript) gives

$$
\frac{d}{d x}\left(u_{g} n_{f}\right)=\phi\left(1-S_{w}\right)\left(k_{1} v_{g}^{1 / 3} v_{w}-k_{-1} v_{g} n_{f}\right) .
$$

Substituting the expressions of the interstitial velocities, we obtain the following:

$$
\frac{d}{d x}\left(u_{g} n_{f}\right)=\phi\left(1-S_{w}\right)\left(k_{1} \frac{u_{g}^{1 / 3}}{\left(\phi X_{f}\left(1-S_{w}\right)\right)^{1 / 3}} \frac{u_{w}}{\phi S_{w}}-k_{-1} \frac{u_{g}}{\phi X_{f}\left(1-S_{w}\right)} n_{f}\right)
$$


where to simplify expressions in the equation, we define $a, b$, and $c$ as

$$
\begin{aligned}
& a=\phi X_{f}\left(1-S_{w}\right), \\
& b=\phi S_{w}, \\
& c=\phi\left(1-S_{w}\right) .
\end{aligned}
$$

The equation is then written as:

$$
\frac{d}{d x}\left(u_{g} n_{f}\right)=c\left(k_{1} \frac{u_{g}^{1 / 3}}{a^{1 / 3}} \frac{u_{w}}{b}-k_{-1} \frac{u_{g}}{a} n_{f}\right)
$$

Assuming that the ideal gas law holds and that the gas velocity in the inlet and outlet are related $\left(P_{g} u_{g}=P_{g L} u_{g L}\right)$, the equation then becomes

$$
\frac{d}{d x}\left(\frac{u_{g L} P_{g L}}{P_{g}} n_{f}\right)=\frac{c k_{1}}{a^{1 / 3} b}\left(\frac{u_{g L} P_{g L}}{P_{g}}\right)^{1 / 3} u_{w}-\frac{c k_{-1}}{a}\left(\frac{u_{g L} P_{g L}}{P_{g}}\right) n_{f} .
$$

Nondimensionlizing the gas-phase pressure by imposed backpressure $\left(P_{g L}\right)$ at the outlet to obtain the dimensionless gas-phase pressure $\left(\bar{P}_{g}=P_{g} / P_{g L}\right)$, we can write:

$$
\begin{aligned}
u_{g L} \frac{d}{d x}\left(\frac{n_{f}}{\bar{P}_{g}}\right) & =\frac{c k_{1} u_{g L}^{1 / 3} u_{w}}{a^{1 / 3} b}\left(\frac{1}{\bar{P}_{g}}\right)^{1 / 3}-\frac{c k_{-1} u_{g L}}{a}\left(\frac{1}{\bar{P}_{g}}\right) n_{f} \\
\frac{d}{d x}\left(\frac{n_{f}}{\bar{P}_{g}}\right) & =\frac{c k_{1} u_{g L}^{-2 / 3} u_{w}}{a^{1 / 3} b}\left(\frac{1}{\bar{P}_{g}}\right)^{1 / 3}-\frac{c k_{-1}}{a}\left(\frac{1}{\bar{P}_{g}}\right) n_{f} .
\end{aligned}
$$

Expanding the left hand side, we obtain:

$$
\frac{\bar{P}_{g} \frac{d n_{f}}{d x}-n_{f} \frac{d \bar{P}_{g}}{d x}}{\bar{P}_{g}^{2}}=\frac{c k_{1} u_{g L}^{-2 / 3} u_{w}}{a^{1 / 3} b}\left(\frac{1}{\bar{P}_{g}}\right)^{1 / 3}-\frac{c k_{-1}}{a}\left(\frac{1}{\bar{P}_{g}}\right) n_{f} .
$$


Nondimensionlizing the bubble texture and space variable by $n_{f L}$ and $L$, respectively, we arrive at the following ordinary differential equation (ODE)

$$
\frac{d \bar{n}_{f}}{d \bar{x}}=\bar{P}_{g}\left(\frac{c L k_{1} u_{g L}^{-2 / 3} u_{w}}{a^{1 / 3} b n_{f L}}\left(\frac{1}{\bar{P}_{g}}\right)^{1 / 3}-\frac{c L k_{-1}}{a}\left(\frac{1}{\bar{P}_{g}}\right) \overline{n_{f}}\right)+\frac{\bar{n}_{f}}{\bar{P}_{g}} \frac{d \bar{P}_{g}}{d \bar{x}}
$$

or, more simply

$$
\frac{d \bar{n}_{f}}{d \bar{x}}=\frac{c L k_{1} u_{g L}^{-2 / 3} u_{w}}{a^{1 / 3} b n_{f L}} \bar{P}_{g}{ }^{2 / 3}-\frac{c L k_{-1}}{a} \bar{n}_{f}+\frac{\bar{n}_{f}}{\bar{P}_{g}} \frac{d \bar{P}_{g}}{d \bar{x}}
$$

that describes how the dimensionless bubble texture/density $\left(\overline{n_{f}}\right)$ changes along the dimensionless domain length $(\bar{x})$.

The second ODE we obtain describes the gas-phase pressure along the domain length. Our starting point is a modified version of $1 D$ multiphase Darcy's law

$$
u_{g}=-K \frac{k_{r g}^{f}}{\mu_{g}^{f}} \frac{d P_{g}}{d x}
$$

where the superscripts $f$ in the gas relative permeability and gas viscosity signify that both functions are adjusted to account for foam. The apparent viscosity of gas in the presence of foam is assumed to be a function of the bubble texture and gas velocity in accordance with literature studies ${ }^{1}$

$$
\mu_{g}^{f}=\mu_{g}+\alpha \frac{n_{f}}{u_{g}^{1 / 3}}
$$

where $\alpha$ is proportionality constant that takes into account the bubble-drag effects. We eliminate the first term on the right hand side of Eq. 7 and simply write

$$
\mu_{g}^{f}=\alpha \frac{n_{f}}{u_{g}^{1 / 3}}
$$

because experimental data in the literature show that the gas apparent viscosity is orders of magnitude (10-1000) larger in the presence of foam. Furthermore, the gas relative perme- 
ability is modified in the presence of foam to be

$$
k_{r g}^{f}=k_{r g}^{0} X_{f}^{3}\left(1-S_{w d}\right)^{3}
$$

where $k_{r g}^{0}$ is the gas end-point relative permeability, $X_{f}$ is the flowing foam fraction, and $S_{w d}$ is the reduced water saturation and is defined as

$$
S_{w d}=\frac{S_{w}-S_{w c}}{1-S_{w c}}
$$

We assume a Corey-like relationship for the gas relative permeability. Combining Eqs. 6-8 and using the gas ideality assumption stated above, we obtain

$$
u_{g}=-K \frac{k_{r g}^{0} X_{f}^{3}\left(1-S_{w d}\right)^{3}}{\alpha \frac{n_{f}}{u_{g}^{1 / 3}}} \frac{d P_{g}}{d x}
$$

or we can write

$$
u_{g}^{2 / 3}=-K \frac{k_{r g}^{0} X_{f}^{3}\left(1-S_{w d}\right)^{3}}{\alpha n_{f}} \frac{d P_{g}}{d x}
$$

which means that we are now ready to write the second ODE

$$
\frac{d P_{g}}{d x}=-\frac{\left(\frac{u_{g L} P_{g L}}{P_{g}}\right)^{2 / 3} \alpha n_{f}}{K k_{r g}^{0} X_{f}^{3}\left(1-S_{w d}\right)^{3}}
$$

or after nondimensionalizing $P_{g}, n_{f}$ and $x$ by $P_{g L}, n_{f L}$, and $L$ we obtain

$$
\frac{d \bar{P}_{g}}{d \bar{x}}=\frac{u_{g L}^{2 / 3} \alpha L n_{f L}}{P_{g L} K k_{r g}^{0} X_{f}^{3}\left(1-S_{w d}\right)^{3}} \bar{n}_{f} \bar{P}_{g}^{-2 / 3}
$$

\section{Model Verification with Experimental Data}

In this section, we verify our steady-state population balance model against existing experimental data. We verify three outcomes of the developed model: the pressure drop across the 
porous medium, the bubble density profile obtained at steady-state, and the flowing foam fraction chosen to match the experimental data. For the first two, we use the steady-state measured pressure drop and the measured steady-state bubble texture along the core of Chen et al.. ${ }^{2}$ We verify the predictions of the flowing foam fraction against those measured by Tang and Kovscek ${ }^{3}$ at varying gas velocities.

\section{Pressure Drop and Bubble Density Profiles}

The literature is full of experimental steady-state pressure drop data but it is difficult to find a study that measures the bubble density profile along the core at steady-state. Fortunately, Chen et al. ${ }^{2}$ sampled the bubble texture along their core length. For this reason, we use their data to verify the model predictions of the pressure drop and bubble density profiles.

Table 1 lists the two-phase and the steady-state population balance parameters used to make our predictions for this case. Figures $1 \mathrm{a}$ and $1 \mathrm{~b}$ show the match between predicted and experimental data. The predicted pressure profile tracks well the measured data but cannot capture the entrance region flatness seen in the measured data. The maximum difference between experimental and predicted pressure data is $4 \mathrm{kPa}$ near $x_{D}=0.2$. The predicted bubble density profile is much smoother than the measured data. Measurements show variation in the measured bubble density especially in the downstream regions closer to the outlet. On the other hand, the trend of both expremental and predicted bubble density profiles agree well. The maximum difference between experimental and predicted bubble density is approximately $120 \mathrm{~mm}^{-3}$ and is found around $x_{D}=0.91$. The bubble texture profile is by definition harder to predict because of two reasons. First, the experimental data accuracy is questionable because to measure the bubble texture, sampling ports must be used. That, in turn, might result in a somewhat different texture than actually is present at the moment of the measurement inside the porous medium. The other reason is that the data is somewhat scattered especially after 0.4 dimensionless distance and is hard to match. Our prediction of the bubble texture is also dictated by the $n_{f L}$ value we choose although 
the overall shape of the bubble texture profile is controlled by the Damköhler numbers of generation and coalescence.

Table 1: Model parameters for foam flow simulation with the steady-state population balance method that are used throughout this paper.

\begin{tabular}{|c|c|c|c|c|}
\hline \multicolumn{5}{|c|}{ Two-phase flow parameters } \\
\hline \multirow[t]{2}{*}{ Parameter } & & Value & & \multirow[t]{2}{*}{ Units } \\
\hline & Chen et al. ${ }^{2}$ & Tang and Kovscek ${ }^{3}$ & Current paper & \\
\hline$K$ & 0.3 & 0.74 & 0.55 & \multirow[t]{4}{*}{$\mu \mathrm{m}^{2}$} \\
\hline$\phi$ & 0.18 & 0.207 & 0.25 & \\
\hline$k_{r g}^{0}$ & 1.0 & 1.0 & 1.0 & \\
\hline$S_{w}$ & 0.53 & measured & estimated & \\
\hline$u_{g L}$ & $1.3 \times 10^{-5}$ & imposed & imposed & \multirow{2}{*}{$\begin{array}{l}\mathrm{m} / \mathrm{sec} \\
\mathrm{m} / \mathrm{sec}\end{array}$} \\
\hline$u_{w}$ & $1.45 \times 10^{-6}$ & $2.2 \times 10^{-6}$ & $4.1 \times 10^{-6}$ & \\
\hline$p_{g L}$ & $2.0 \times 10^{6}$ & $8.27 \times 10^{5}$ & $7.55 \times 10^{5}$ & \multirow[t]{2}{*}{$\mathrm{Pa}$} \\
\hline$S_{w c}$ & 0.38 & 0.25 & 0.25 & \\
\hline \multicolumn{5}{|c|}{ Population balance parameters } \\
\hline \multirow[t]{2}{*}{ Parameter } & & Value & & \multirow[t]{2}{*}{ Units } \\
\hline & Chen et al. ${ }^{2}$ & Tang and Kovscek ${ }^{3}$ & Current paper & \\
\hline$k_{1}$ & $1.95 \times 10^{15}$ & $1.75 \times 10^{16}$ & $1.15 \times 10^{15}$ & $\mathrm{~m}^{-13 / 3} \mathrm{~s}^{1 / 3}$ \\
\hline$k_{-1}$ & 10 & 110 & 45 & $\mathrm{~m}^{-1}$ \\
\hline$n_{f L}$ & $4.0 \times 10^{11}$ & measured & $4.0 \times 10^{11}$ & $\mathrm{~m}^{-3}$ \\
\hline$\alpha$ & $8.4 \times 10^{-19}$ & $3.48 \times 10^{-17}$ & $4.1 \times 10^{-16}$ & $\mathrm{~Pa}-\mathrm{s}^{2 / 3}-\mathrm{m}^{10 / 3}$ \\
\hline$X_{f}$ & 0.22 & varying & varying & \\
\hline
\end{tabular}

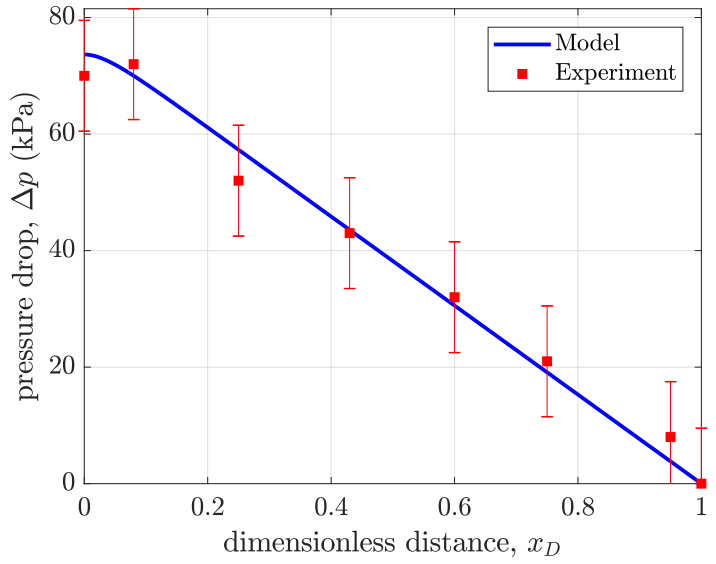

(a)

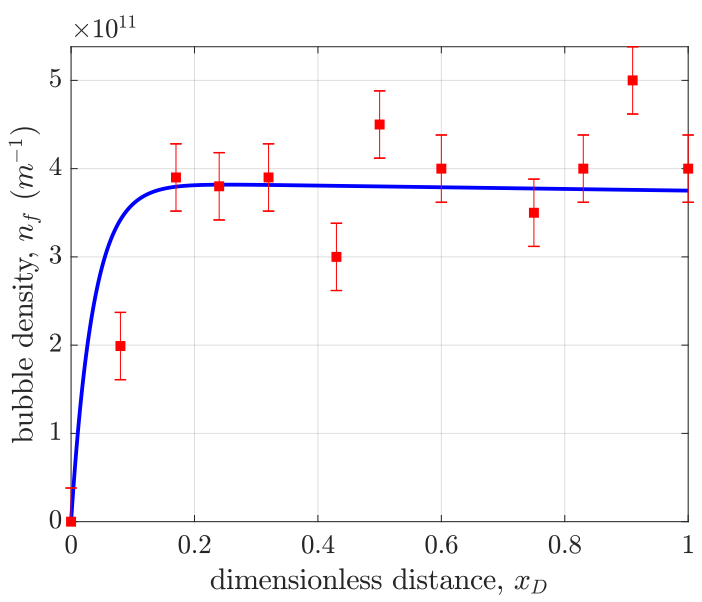

(b)

Figure 1: Verification of steady-state pressure drop and bubble density predictions. Model results are shown in blue, while experimental results of Chen et al. ${ }^{2}$ are shown in red dots with error bars. 


\section{Flowing Foam Fraction}

One important parameter that is still not yet verified is the flowing foam fraction assigned to match an experiment. There are only a few experimental studies that focus on measuring the flowing foam fraction in flow scenarios ${ }^{3-5}$ even though it is one of the most important parameters used to match experimental data. The flowing foam fraction directly affects the gas relative permeability. We chose to match the data of Tang and Kovscek ${ }^{3}$ who used tracers to measure the flowing foam fraction. They also report the resulting pressure gradient and effluent bubble texture. It is important to note that they only report the overall steady-state pressure gradient and the average water saturation in each experiment. Therefore, the shape of the pressure gradient profile is unknown.

During the matching procedure, we used any measured or imposed parameters as inputs. As listed in Table 1, the water saturation and the effluent bubble texture were measured while the gas Darcy velocity at the experimental backpressure was reported. Therefore, these parameters are set for each experiment. The foam kinetics parameters, $k_{1}$ and $k_{-1}$, as well as the viscosity proportionality constant were fixed during matching all experiments. In order to obtain these, we matched the last experiment of Tang and Kovscek. ${ }^{3}$ Importantly, the ratio of $k_{1} / k_{-1}$ sets the bubble texture and fixes the dependence between these two parameters. ${ }^{2,6}$ Therefore, the only parameter that varies in the experiments is the flowing foam fraction, $X_{f}$, and it is adjusted to match the measured history.

Figures $2 \mathrm{a}$ and $2 \mathrm{~b}$ report the predicted versus the measured pressure gradient and effluent bubble density, respectively. The pressure gradient match is excellent with a maximum difference between the predicted and measured data of $134 \mathrm{kPa} / \mathrm{m}$ when the gas velocity is $30.2 \mathrm{~m} /$ day. The effluent bubble texture match is good when the gas velocity is large. The average difference between the predicted and the measured effluent bubble density for the three smallest gas velocities employed in Tang and Kovscek's ${ }^{3}$ experiments is $400 \mathrm{~mm}^{-3}$ and the difference is $100 \mathrm{~mm}^{-3}$ for the remaining employed gas velocities. Figure 3 reports the measured average water saturation, which represents the same value used in the model to 
predict the measured parameters. Figure 4 plots the predicted flowing foam fraction for each experiment and compares these values with the experimental data. The model overestimates the measured $X_{f}$ at small gas velocities and underestimates the measured values at large gas velocities but captures the overall trend of increasing $X_{f}$ with increasing pressure gradient of the system. The average difference between the predicted and measured $X_{f}$ values is $6 \%$. Even with the mismatches, the model seems to be in good agreement with regards to the general trend and the trend is more important for our purposes.

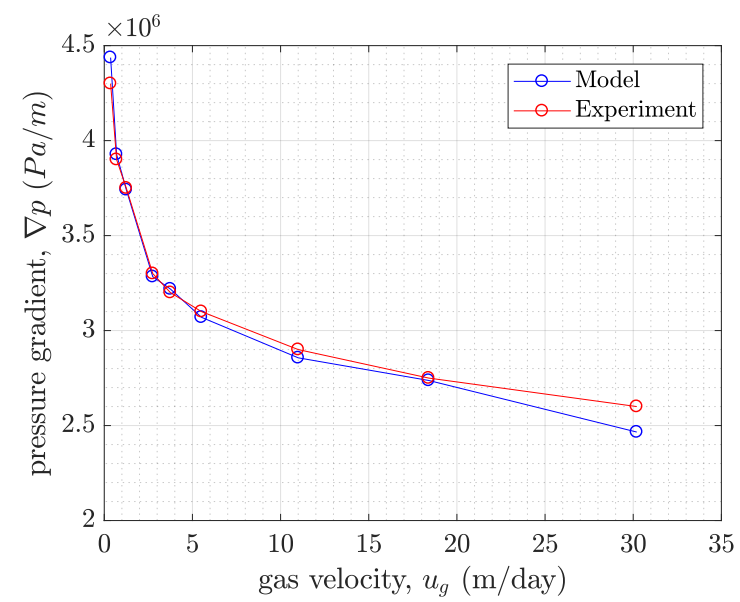

(a)

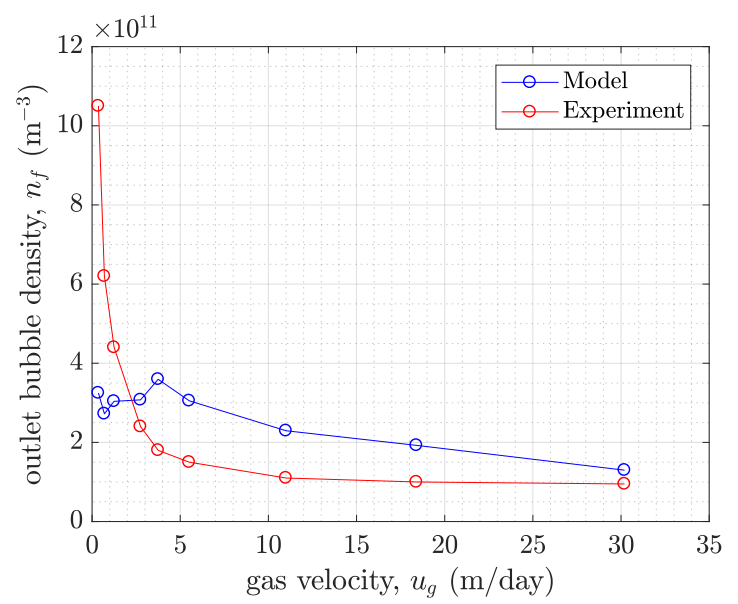

(b)

Figure 2: Verification of steady-state (a) pressure gradient predictions and (b) outlet bubble density predictions. Model results are shown in blue, while experimental results of Tang and Kovscek $^{3}$ are shown in red. 


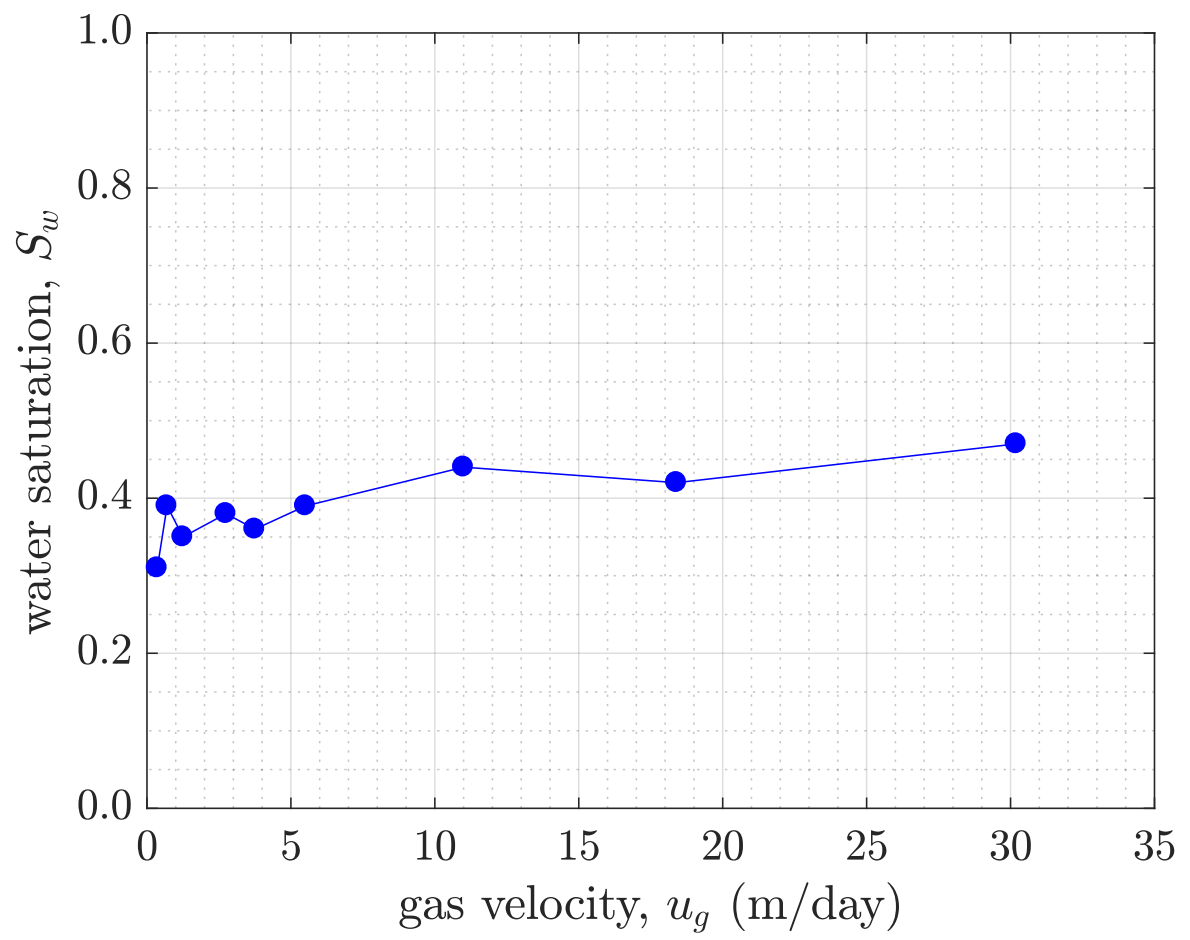

Figure 3: Measured steady-state water saturation of Tang and Kovscek. ${ }^{3}$ 


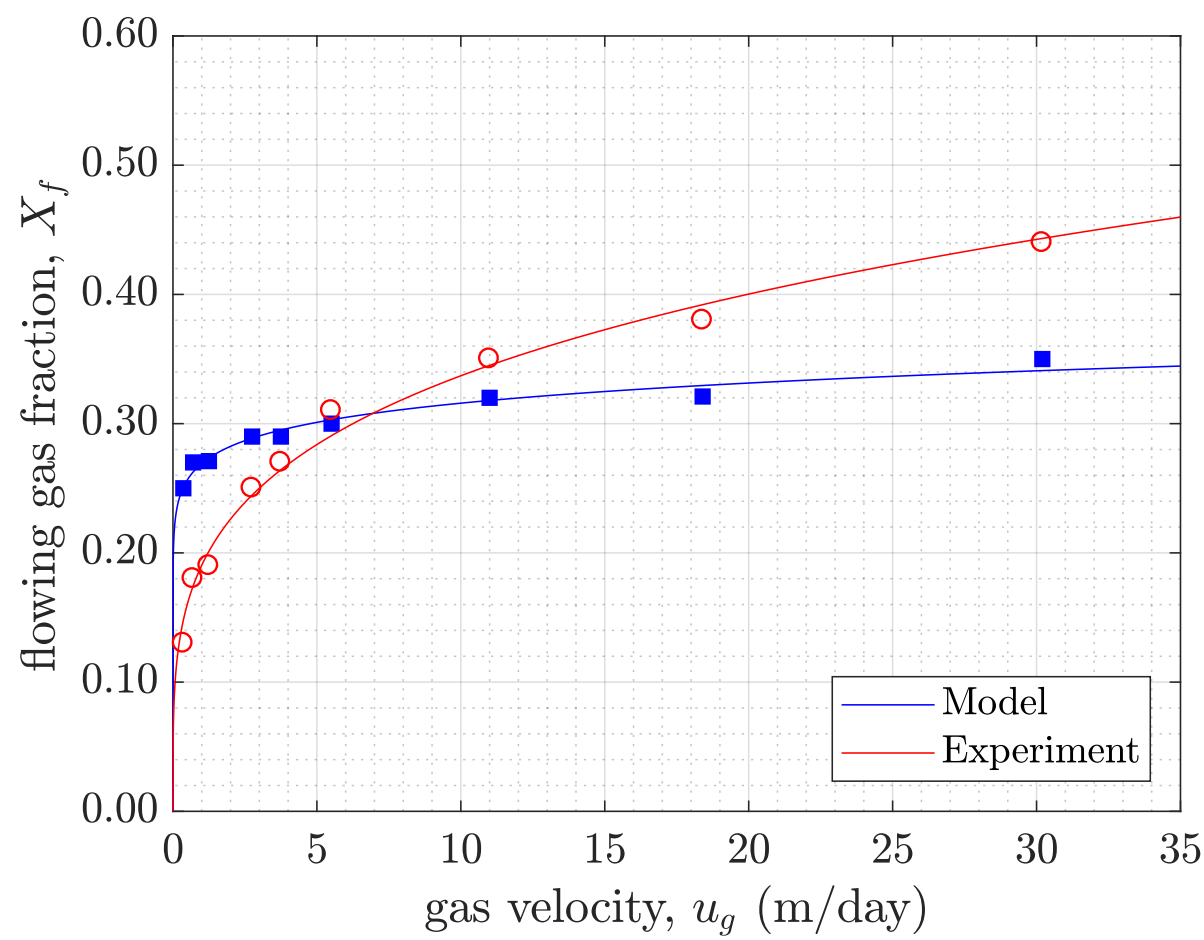

Figure 4: Comparison between steady-state flowing foam fraction predictions and experimental results. Model results are shown in blue, while experimental results of Tang and Kovscek $^{3}$ are shown in red.

\section{Dimensionless Parameters Effect on Output Parameters}

In this section, we examine the sensitivity of the output parameters of the steady-state population balance model to the three dimensionless parameters: the generation Damköhler number $\left(D a_{1}\right)$, the coalescence Damköhler number $\left(D a_{-1}\right)$, and the compressiblility factor $(\kappa)$. Most of the plots show the average dimensionless pressure drop $\left(\Delta \tilde{p}_{g}\right)$, the average dimensionless bubble density/texture $\left(\tilde{n}_{f_{a v g}}\right)$, and the predicted dimensionless effluent bubble density $\left(\tilde{n}_{f L_{\text {pred }}}\right)$.

Figure 5 summarizes the effect of the three dimensionless numbers on the listed three parameters. The figures show 30 slices of pressure maps, each slice has a constant $\kappa$. As expected, the dimensionless pressure drop is larger when generation dominates and fades away as generation and coalescence equilibrate. Interestingly, the pressure drop is more 
proportional to the dimensionless average dimensionless bubble density in the system than the effluent dimensionless bubble density.

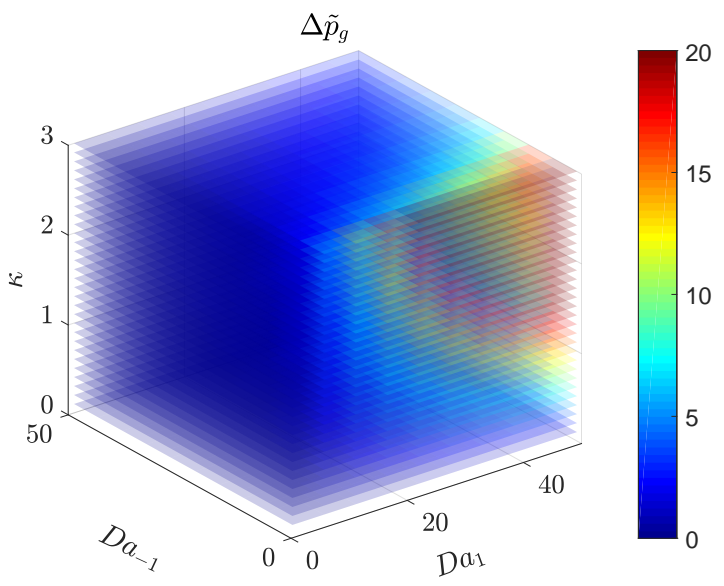

(a)

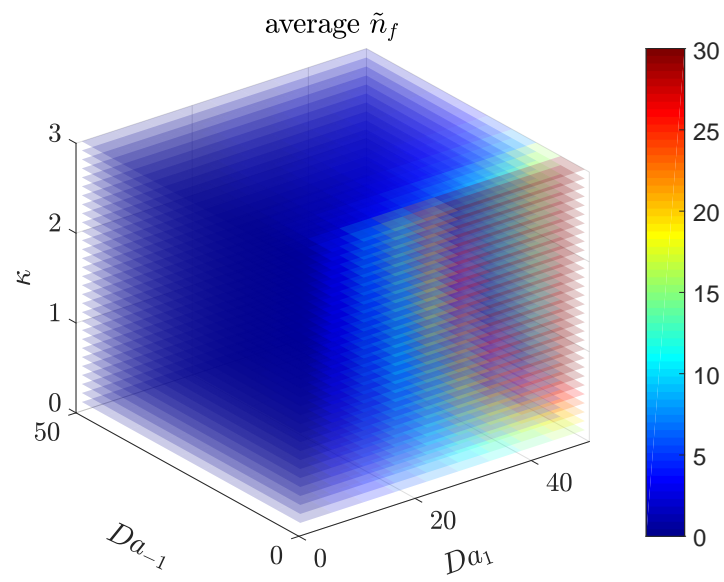

(b)

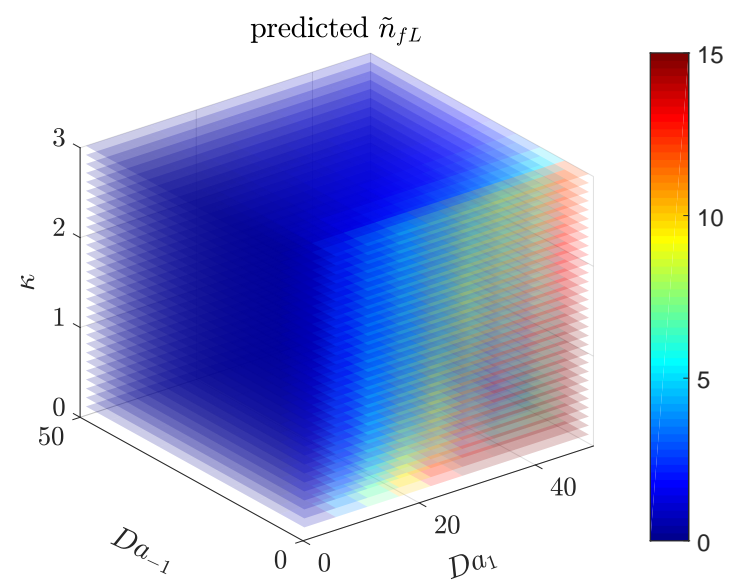

(c)

Figure 5: Sensitivity to various combinations of all three dimensionless parameters. The generation Damköhler number, $D a_{1}$, the coalescence Damköhler number, $D a_{-1}$, and the compressiblility factor, $\kappa$.

Figure 6 shows the effect of varying the ratio of the Damköhler numbers ratio, $D a_{1} / D a_{-1}$, and the compressiblility factor, $\kappa$, on the three dimensionless parameters. Similar to the trend we pointed out in Fig. 5, the dimensionless pressure drop seem to be proportional to the average dimensionless bubble density in the core and not necessarily proportional to the effluent dimensionless bubble density. We notice that to achieve a large pressure drop, not only does the generation Damköhler number need to be large but also the compressiblility 
factor.

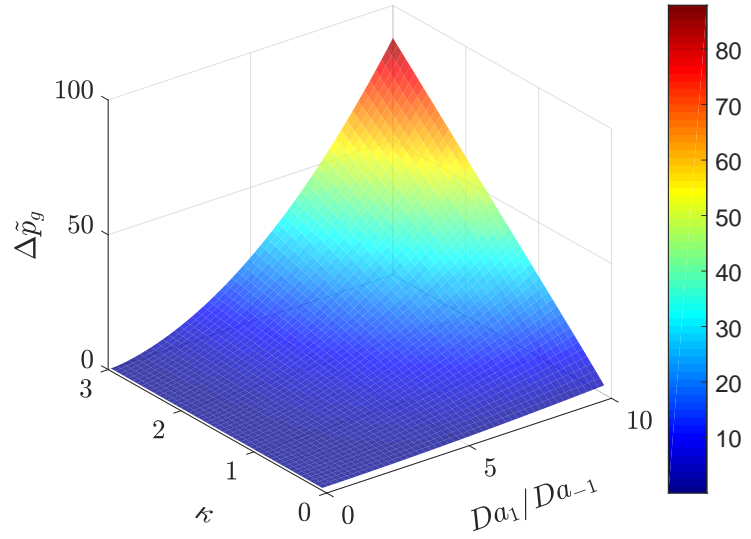

(a)

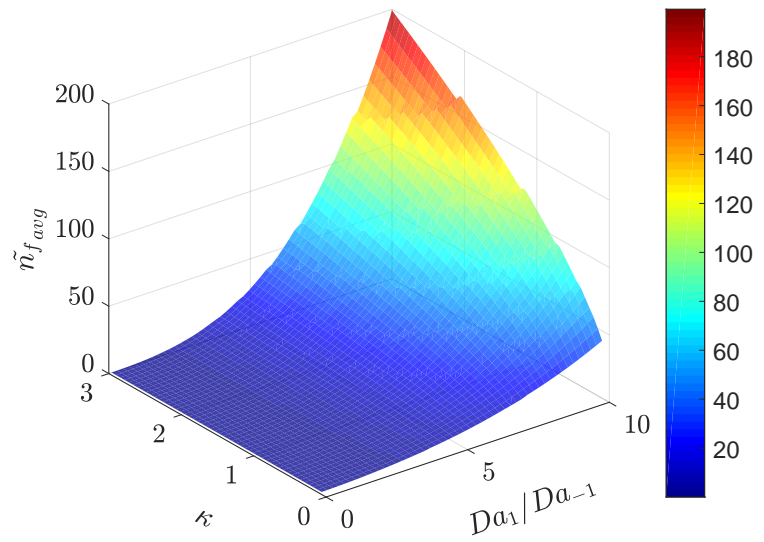

(b)

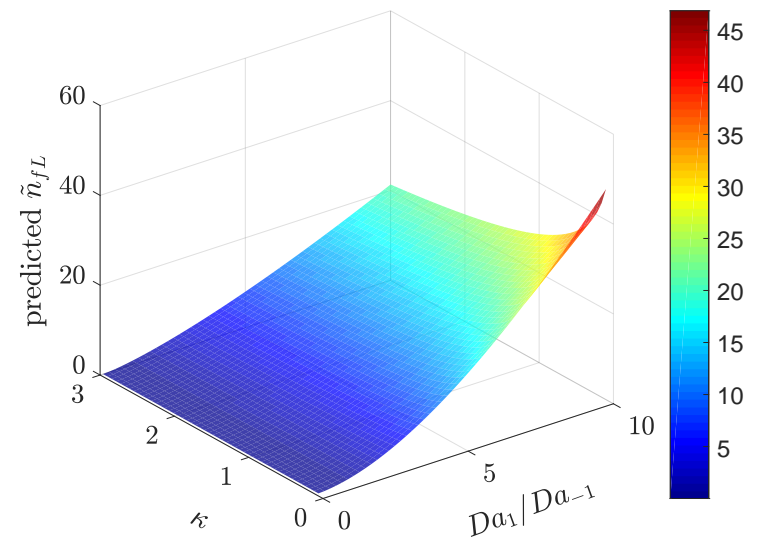

(c)

Figure 6: Sensitivity to various combination of Damköhler numbers ratio $D a_{1} / D a_{-1}$ and $\kappa$ values.

The net generation region is the region where generation overcomes coalescence or where the bubble texture is getting finer. It is not so clear how to define a net generation region. Therefore, we proceed by assuming that the generation overtakes coalescence if the dimensionless bubble texture, $\tilde{n}_{f}$, is larger than the outlet dimensionless bubble texture, $\tilde{n}_{f L}$ which is 1 . Figure 7 a shows the dimensionless distance where the maximum value of $\tilde{n}_{f}$ appears given that it is larger than 1. Examining this figure alone to delineate generation is incorrect. To form a complete picture of the net generation in the system, we also need to determine how fast does the dimensionless bubble texture reaches 1 . Figure $7 \mathrm{~b}$ shows the 
nearest dimensionless distance from the inlet where the dimensionless bubble texture equals or exceeds 1 . When $D a_{1}$ is large, we expect it to dominate the generation and, therefore, the plot shows that it takes very little distance for the dimensionless bubble texture to reach 1. On the other hand, when $D a_{-1}$ is dominating, the dimensionless bubble texture never reaches 1 . Now, we define a net generation coefficient (NGC) that takes into account both of these factors

$$
\mathrm{NGC}=\frac{x_{D} \text { of } \max \tilde{n}_{f}}{x_{D} \text { of first } \tilde{n}_{f} \geq 1}
$$

where we divide the surface in Fig. 7a over the surface in Fig. 7b. Figure 7c shows the shape of the net generation coefficient. When the generation Damköhler number is large, NGC is large but decreases sharply until about coalescence Damköhler number of 20 where it levels off. The plot shows that net generation in porous media is a delicate balance between generation and coalescence events that depend on many variables and could be interpreted many different ways. 


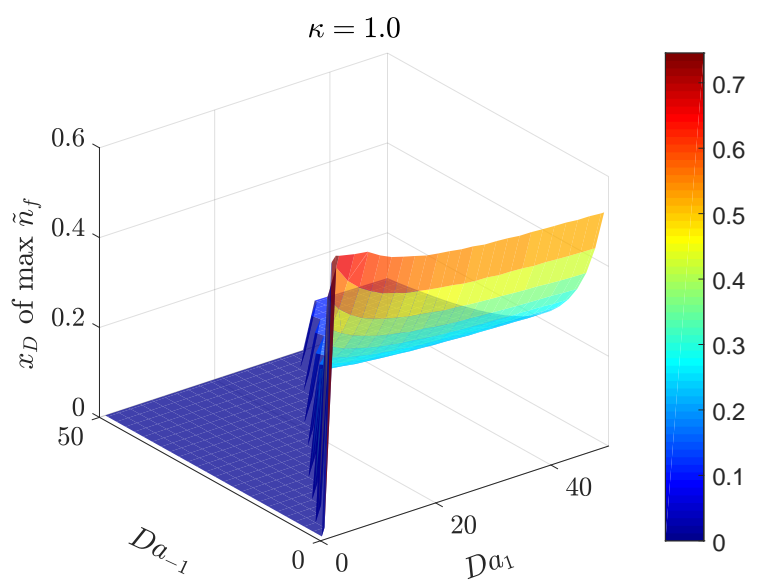

(a)

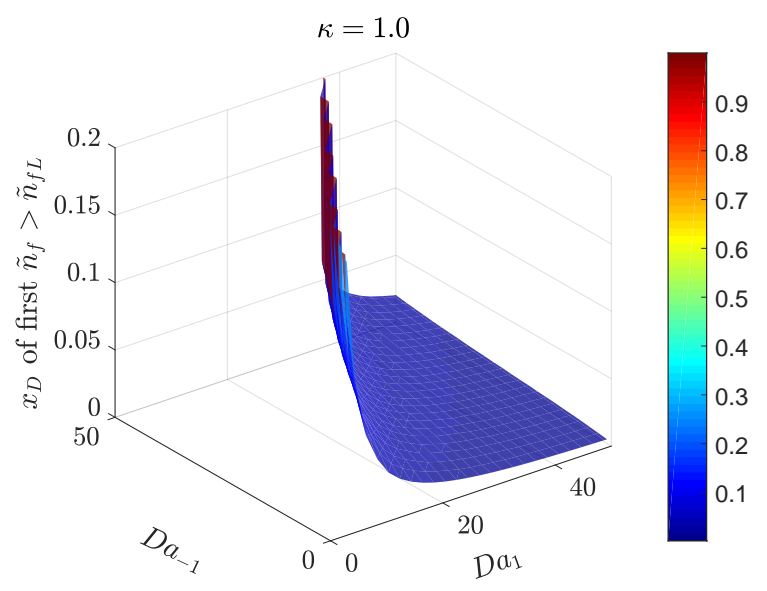

(b)

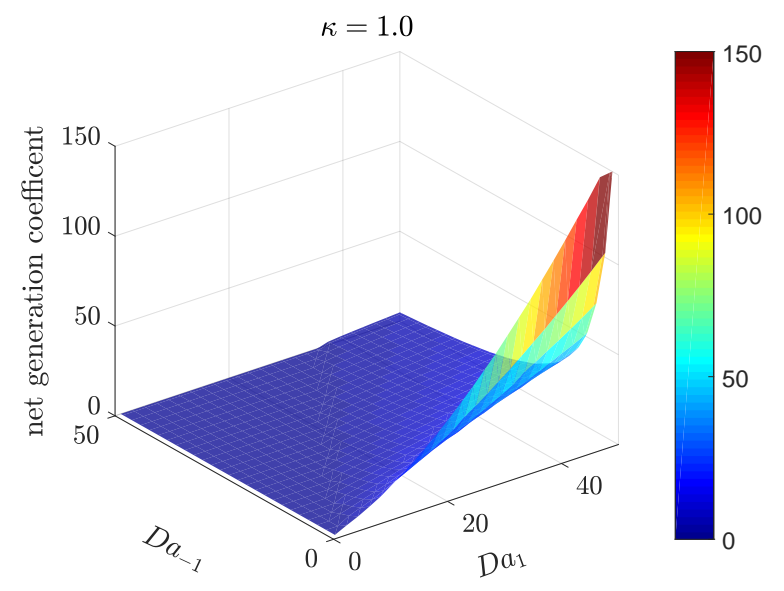

(c)

Figure 7: Net foam generation region sensitivity while varying the generation and coalescence Damköhler numbers, $D a_{1}$ and $D a_{-1}$, and keeping the compressiblility factor, $\kappa$, constant.

\section{References}

(1) Hirasaki, G. J.; Lawson, J. B. Mechanisms of foam flow in porous media: apparent viscosity in smooth capillaries. SPE J 1985, 25, 176-190.

(2) Chen, Q.; Kovscek, A. R.; Gerritsen, M. Modeling foam displacement with the localequilibrium approximation: theory and experimental verification. SPE Journal 2010, $15,171-183$. 
(3) Tang, G.-Q.; Kovscek, A. R. Trapped gas fraction during steady-state foam flow. Transport in porous media 2006, 65, 287-307.

(4) Gillis, J. V.; Radke, C. J. A dual gas tracer technique for determining trapped gas saturation during steady foam flow in porous media. SPE Annual Technical Conference and Exhibition. 1990.

(5) Nguyen, Q. P.; Rossen, W. R.; Zitha, P. L. J.; Currie, P. K. Determination of gas trapping with foam using X-ray computed tomography and effluent analysis. SPE Journal 2009, 14, $222-236$.

(6) Ettinger, R. A.; Radke, C. J. The influence of texture on steady foam flow in berea sandstone. 1989, 